\title{
Photographier Zermatt : les pratiques photographiques des touristes à l'épreuve du numérique
}

Capturing Zermatt: Tourist's photographic practices in the digital era

\section{Ellina Mourtazina}

\section{(2) OpenEdition}

Journals

Édition électronique

URL : http://journals.openedition.org/tourisme/2148

DOI : 10.4000/tourisme.2148

ISSN : 2492-7503

Éditeur

Éditions touristiques européennes

Référence électronique

Ellina Mourtazina, "Photographier Zermatt : les pratiques photographiques des touristes à l'épreuve du numérique », Mondes du Tourisme [En ligne], 15 | 2019, mis en ligne le 01 juin 2019, consulté le 30 octobre 2019. URL : http://journals.openedition.org/tourisme/2148; DOI : 10.4000/tourisme.2148

Ce document a été généré automatiquement le 30 octobre 2019

\section{cc) (ㅇ)}

Mondes du tourisme est mis à disposition selon les termes de la licence Creative Commons Attribution

- Pas d'Utilisation Commerciale - Pas de Modification 4.0 International. 


\title{
Photographier Zermatt : les pratiques photographiques des touristes à l'épreuve du numérique
}

Capturing Zermatt: Tourist's photographic practices in the digital era

\author{
Ellina Mourtazina
}

\section{Introduction}

1 La pratique photographique a acquis, au cours de l'histoire du phénomène touristique, une place prédominante et représente aujourd'hui une activité emblématique des vacanciers. Dès la seconde moitié du XIX siècle, alors que l'industrie naissante des loisirs développe des technologies de mobilité et rend les lieux lointains physiquement accessibles, les images photographiques quant à elles viennent fragmenter les visions du monde, alimenter un imaginaire de voyage et dicter les prémices d'un regard touristique sur le monde (Urry, 1990). Soutenus par leur développement technologique respectif, mobilité et visualité sont rapidement devenues de puissants moteurs de la modernité et des piliers du tourisme. Dès le lancement du Brownie en 1880 - le premier modèle portatif d'appareil photographique - sous le slogan «Vous appuyez sur le bouton, nous faisons le reste", l'industrie Kodak a réussi son pari de rendre accessible la photographie au plus grand nombre en réduisant au maximum ses fonctionnalités et ses étapes de réalisation. Avec la démocratisation de son emploi, la photographie a progressivement intégré les sphères intimes du quotidien et a engendré une pratique sociale à part entière qui, à travers les époques, s'est ajustée aux développements des dispositifs technologiques des appareils. Dès leur genèse chez Kodak, les appareils de prise de vue, dans leurs successives versions analogiques, ont principalement répondu à l'unique fonction de capturer par image les réalités visuelles du monde.

2 Toutefois, au tournant des années 2000, avec l'arrivée du numérique et dans un contexte de globalisation et de mobilité accrue, les appareils photographiques se sont «hybridifiés » dans leurs fonctionnalités et leurs usages. En devenant numérique, la 
photographie a intégré la sphère des technologies mobiles et son processus de production répond dorénavant à d'autres manières de faire et d'être avec les images. En se séparant de son corps papier et à partir de son essence de code binaire, l'image numérisée est visionnée instantanément, modifiée, partagée, multipliée ou supprimée. Ces nouvelles modalités ont conduit à des renégociations du rapport entre l'espace photographié, la matérialité du dispositif technologique et l'engagement corporel et émotionnel de l'individu - en somme, à une modification des manières de faire de la photographie. De plus, en étendant la photographie à des moments jusque-là peu propices à cette pratique, ces potentialités nouvelles du numérique ont également changé la constellation (des espaces et des temporalités) à travers laquelle les images de vacances sont appréhendées. La pratique photographique s'est, d'une part, étendue et densifiée au cours des temporalités qui précèdent et succèdent le séjour et, d'autre part, elle a investi de nouveaux espaces et temporalités durant le séjour touristique. Les salles d'attente, le temps passé dans le train, les soirées à l'hôtel, l'attente dans les restaurants - des instants souvent jugés non saillants pour le regard touristique - sont devenus des instants photographiques, au cours desquels l'image est négociée, appropriée ou rejetée. En questionnant les rapports de proximité et de distance, du quotidien et hors-quotidien, la nature hybride de la photographie numérique conduit à repenser les temporalités et spatialités qui soutiennent les différentes formes de sociabilité et de coprésence.

3 Le numérique conduit également à une renégociation du rapport à la matérialité photographique. Si, traditionnellement, la photographie a été regardée comme un objet constitué de deux facettes non séparables - à savoir l'objet physique (papier) et l'image représentée -, cette lecture de la culture matérielle photographique est à remettre en jeu. Comme le souligne Sassoon (2004), aujourd'hui la présence physique au monde de l'image se manifeste sous différentes formes et le propre de sa matérialité numérique est de naviguer entre les régimes du visible et de l'invisible, du tactile et de l'impalpable, de bénéficier d'une pérennité et de prendre des formes stables aussi bien que mobiles et à courte durée de vie. Ainsi, repenser le rapport à l'objet et extraire la photographie d'une conception dualiste matériel/immatériel, où le matériel est vu uniquement sous le prisme de sa substance physique, semble être une étape indispensable pour l'étude des pratiques photographiques.

4 S'il existe une riche littérature en sciences humaines traitant de la photographie touristique (Crouch et Lübbren, 2003 ; Larsen, 2003 ; Osborne, 2000 ; Schroeder, 2002 ; Taylor, 1994 ; Urry, 2002), la dimension numérique et les changements auxquels elle a conduit sur la manière de vivre l'expérience restent, à ce jour, sous-documentés. Confinée dans un premier temps au registre visuel, la photographie a longtemps été considérée comme l'objet fini d'un processus prescriptif de sélection d'images touristiques, et non en tant que pratique sociale vivante imbriquant corporalité, espace et technologie. De plus, approchées comme un procédé mécanique de duplication de la réalité, les temporalités de la performance photographique étaient alors réduites à un seul instant, celui de la capture d'image, et non regardées comme un ensemble de micro-pratiques s'étendant sur des temporalités plus longues : tant avant, pendant, qu'après le voyage. Si les études plus récentes mettent davantage l'accent sur la dimension performative et non pré-formative de l'expérience touristique (Larsen, 2004), les manières de faire prenant en compte les potentialités apportées par la dimension numérique restent toujours au second plan de l'analyse. 
5 À travers un travail ethnographique mené auprès de visiteurs de la station suisse de Zermatt, l'objectif de cet article est d'apporter un éclairage sur les différentes dimensions du faire photographique numérique, considéré ici comme un ensemble de pratiques qui associent normes esthétiques, matérialité, numérique, corporéité et espaces. Comme cela va être vu dans la suite de l'articlent, cette notion volontairement large - permet de prendre en compte les processus qui articulent les normativités collectives de l'action aux réalités spécifiques et expérientielles des espace-temps photographiques. À travers une description détaillée des différents microinstants qui séquencent et rythment la pratique photographique numérique des touristes durant leur séjour à Zermatt, nous proposons dans cet article d'appréhender l'expérience photographique non pas uniquement comme une manière de voir - c'est-àdire de poser un regard préconstruit sur les paysages vécus -, mais également comme une manière d'être-au-monde et de faire avec ces éléments du monde environnant. Le questionnement qui sous-tend ce travail est donc de voir comment les touristes s'approprient, négocient et incorporent les nouvelles potentialités numériques dans leurs performances photographiques; et aussi, plus largement, de comprendre quelle place tient dorénavant la pratique photographique au sein de l'expérience de voyage?

Avant d'aller plus loin, il convient à ce stade de préciser l'élément suivant : bien que l'accent de ce travail soit mis sur la dimension numérique, il ne s'agit pas d'inscrire la photographie actuelle dans un paradigme de changement et d'opposition avec «l'ancien » analogique. Qu'elle soit numérique ou digitale, la photographie est un outil de mémoire, d'interrelation, de représentation et d'expression de soi à travers temps et espace (Van House, 2011). Et l'une des raisons qui a conduit à son acceptation dans les usages est sa continuité avec les modalités de l'analogique. En effet, ce passage au numérique a rendu visible les acquis de la pratique photographique analogique tout en révélant les transformations dans les modes de faire. Dans cet article, il s'agit donc de mieux comprendre la manière dont ces deux modes naviguent entre continuités et ruptures. Pour «prendre le pouls » de la pratique photographique actuelle, l'analyse des pratiques et des manières de faire constitue, à notre sens, une porte d'entrée privilégiée pour ne pas tomber dans la dichotomie de l'ancien et du nouveau.

\section{Le faire photographique : entre regards pré-formatifs et gestes performatifs}

7 À partir des années 1970 et l'émergence du tourisme comme objet d'étude des sciences sociales et humaines, la photographie - rapidement identifiée comme pratique inhérente au phénomène touristique (Crang, 1997 ; Sontag, 1977 ; Urry, 1990) - s'est inscrite dans les mêmes courants théoriques et postures idéologiques qui ont façonné les premières recherches de ce champ. Cristallisant d'une part le questionnement sur les divers impacts perpétrés par un tourisme dit "de masse " sur les sociétés visitées (Leite et Graburn, 2010) et se confinant d'autre part à un paradigme visuel (Sontag, 1977, la photographie touristique a été regardée essentiellement comme un produit fini de consommation d'un répertoire visuel de lieux jugés dignes d'intérêt par le regard touristique. Considérée comme une pratique essentiellement du voir, et moins du faire ou du être, l'intérêt a alors porté sur les mécanismes globaux et les forces extérieures telles que les images produites par l'industrie du tourisme - agissant sur les comportements des touristes, et non sur l'analyse des pratiques sociales vivantes en 
train de se faire. Dans ce contexte, l'insensibilité vis-à-vis des manières de faire de la photographie s'inscrit dans un désintérêt plus général pour la personne du touriste et pour son engagement corporel et émotionnel au monde. Dans les premiers écrits scientifiques, le touriste - dépossédé de sa corporéité au profit de l'immatérialité du regard - a été considéré en tant qu'élément d'un groupe reproduisant à l'identique les clichés d'un monument, d'un lieu ou d'un paysage (Crouch et Desforges, 2003). La pratique photographique servait davantage comme porte d'entrée pour l'analyse de normativités collectives et de prescriptions sociales. Au cours des dernières décennies toutefois, prenant le contrepied de ce paradigme représentationnel, tout un pan de l'intérêt scientifique s'est déplacé vers les expériences sensibles et immédiates des touristes photographes - c'est-à-dire sur les manières dont les individus s'engagent, s'approprient, négocient les éléments qui forment la réalité de leurs mondes vécus dans un contexte touristique (Haldrup et Larsen, 2012; Robinson et Picard, 2009; Scarles, 2009). L'intérêt pour le regard préformé du touriste a ainsi laissé une plus grande place à l'action performative et à l'engagement sensoriel de l'être dans la situation photographique. Comme l'a mentionné Crang (1997), faire de la photographie consiste à prendre part à la réalité vécue plutôt qu'à la refléter.

8 En ayant conscience des apports de chacune de ces deux approches, il s'agira dans cet article non pas de se positionner en faveur de l'une ou de l'autre, mais de les articuler. À notre sens, la pratique photographique ne renvoie pas qu'aux actions pré-formatives ou performatives mais résulte de la jonction entre les deux. L'expérience photographique n'est pas uniquement un acte de reproduction machinale de paysages touristiques anticipés. D'un autre côté, il n'est pas possible de ne traiter la pratique photographique qu'en termes d'immédiateté de l'expérience sensorielle, comme une relation "pure» et "innocente» au monde. Au moment d'arriver sur les lieux photographiques, les touristes ne sont pas des "pages blanches ", dans la mesure où ils mobilisent un certain nombre de compétences techniques, spatiales et corporelles, des savoir-faire et des savoir-être, ainsi que certaines représentations des lieux nourries par les connaissances, les imaginaires et les jugements esthétiques (Robinson et Picard, 2009). Ce bagage est négocié et transformé une fois en situation d'action photographique. Afin de prendre en compte ces deux forces motrices, nous articulons la pratique photographique des touristes autour de trois modes d'engagements au monde, à savoir : une manière de voir, d'être et de faire. L'expérience des touristes/ photographes présuppose de poser un regard esthétique, éthique et culturel sur l'objet de son intention (Urry, 1990). Investie d'attentes et d'anticipations, cette manière de pré-voir l'expérience à venir est mise à l'épreuve des aléas des réalités vécues. Les individus se trouvent être présents avec les réalités immédiates et doivent renégocier leurs attentes à travers des actions et des gestes concrets; en somme, à travers l'acte de faire. À notre sens, ce triptyque qui compose le faire photographique permet d'éclairer, à travers la dimension expérientielle de la pratique, l'articulation entre codes sociaux, jugements esthétiques, environnements physiques et réalité immédiate dans laquelle les touristes-photographes sont plongés.

9 Dans cette manière d'envisager la pratique photographique, le corps - en tant que construit social et culturel - est considéré comme un outil majeur d'engagement au monde. Suivant la pensée que l'expérience vécue est créée à travers la corporalité même de la personne qui la vit (Csordas, 1994), le corps est considéré comme un « point d'affordance » (Veijola et Jokinen, 1994) ou comme le «point zéro existentiel ${ }^{1}$ 
(Desjarlais et Throop, 2011) à partir duquel l'expérience photographique est vécue. Par une inscription sociale et culturelle de l'individu, la corporalité permet d'appréhender l'expérience de l'espace environnant et de la matérialité des objets mobilisés. Dans ce rapport à l'espace, les qualités des lieux touristiques ne sont pas uniquement récoltées par l'outil de la caméra en tant que données visuelles, mais sont co-constituées avec et par l'engagement multisensoriel du corps des individus qui performent la photographie.

\section{Zermatt : une station vedette}

Pour aborder la pratique photographique, l'article se base sur une enquête de terrain menée au cours de l'année 2015 à Zermatt, station de montagne située dans les Alpes suisses, qui correspond à ce que l'Équipe MIT nomme un "haut lieu touristique " (2005). Ayant accumulé au fil des décennies un capital symbolique et réputationnel fort, Zermatt est aujourd'hui représentée comme la plus ancienne station touristique de Suisse. Elle est mise en valeur notamment pour : son glorieux passé d'alpinisme, à son apogée lors de la première ascension du mont Cervin en 1865; son infrastructure hôtelière, dont certains bâtiments - comme l'Hôtel Mont Rose et l'Hôtel Mont Cervin se démarquent par l'historicité dont ils sont investis; son interdiction de circulation aux véhicules motorisés; et, enfin, ses boutiques de luxe. Toutes ces caractéristiques, largement mises en avant par les acteurs du tourisme local et international, attirent chaque année des centaines de touristes, et ce tout au long de l'année. Surtout, Zermatt possède sa montagne vedette : le Cervin, qui dans l'imaginaire de nombreux touristes est devenu le symbole de la Suisse.

$11 \mathrm{Si}$, dans son ensemble, le village de Zermatt connaît une haute fréquentation touristique, tous les espaces environnants ne bénéficient pas du même régime de valeur au regard des touristes et certains pôles ou artères de la ville connaissent des flux touristiques plus importants. Dans les environs, le Gornergrat, le Rieffel, le Sunnegga ou le Matterhorn Glacier Paradise constituent des lieux au fort capital symbolique. La vue dégagée sur le Cervin fait de ces lieux des points privilégiés pour la photographie touristique. Conscient de la prolifération internationale d'images de Zermatt capturées sous ces angles, l'office du tourisme de Zermatt répertorie sur sa plateforme internet une dizaine de lieux, au sein et en périphérie de la station, nommés photopoints, introduits par le préambule suivant sur la plateforme internet ${ }^{2}:$ " Les sujets photo de Zermatt font le tour du monde: Cervin, Mont Rose, Rothorn, Riffelsee, Schwarzsee, glaciers, éboulement de Randa, Grand Hôtel, et bien plus encore. Pourquoi ne pas prendre soi-même un tel cliché ?». Les points photographiques proposent de combiner le capital symbolique du Cervin, l'activité de randonnée en montagne et la pratique photographique. Cette stratégie permet notamment de désengorger certains espaces de la station en créant de nouveaux pôles d'attractivité. Mais, surtout, les touristes sont invités à s'approprier et à expérimenter par eux-mêmes ces paysages vedettes de Zermatt. C'est à l'emplacement de ces points photographiques qu'a été mené le travail de terrain ethnographique, au cours de l'année 2015. Il a été constitué par l'observation participante d'individus s'adonnant à la pratique photographique et par 46 entretiens semi-directifs. 


\section{Photographier Zermatt}

\section{Trouver la bonne place : stratégies de placement dans l'espace photographique}

L'observation de touristes s'adonnant à des performances photographiques nous a permis de voir qu'au moment de capturer la photographie, les savoir-faire et savoirêtre, les représentations des lieux, les attentes et les anticipations sont remis en jeu. En dépit de ses expériences photographiques antérieures, arrivé sur un nouveau lieu touristique, l'individu procède à des micro-ajustements et adaptations de ses compétences par rapport à la réalité du lieu, à ses outils photographiques et aux autres touristes présents sur place.

Les photopoints étant souvent bondés de touristes ayant pour finalité de s'immortaliser en face du Cervin, les personnes doivent faire avec et en fonction de leurs semblables. Étant profondément relationnelle, cette coprésence devient ce que Lussault (2009) nomme une "épreuve spatiale ", dans laquelle les individus doivent gérer la séparation qui existe entre eux. Se mettent dès lors en place diverses stratégies de placement et d'évitement pour trouver la "bonne place » et capturer « la bonne photo » en fonction de ses préférences esthétiques, des corps d'autrui, de ses capacités techniques et de ses connaissances technologiques de l'appareil. Durant ces micro-instants de grande proximité imposée, la dimension affective et émotionnelle joue également un grand rôle dans la manière de gérer ces espaces-événements. Certains s'irritent, d'autres prennent leur mal en patience, certains abandonnent le projet de faire un cliché, d'autres encore "forcent le destin " pour se placer aux premières loges. Afin de ne pas gâcher la qualité de sa propre photographie en laissant figurer un inconnu dans sa mise en scène, mais également pour ne pas gâcher le cliché des autres dans cette chorégraphie, les déplacements des touristes sont cadencés par des temps d'attente et d'action. Dès lors que certaines places sont reconnues informellement comme des emplacements idéaux pour la mise en scène photographique, apparaît un conflit d'usage tacite entre les personnes qui les utilisent de façon différente. On demande aux personnes qui restent un temps jugé «trop long " par les autres touristes devant ces espaces sans utiliser leur appareil de se déplacer. Ainsi, les manières d'occuper l'espace et les conditions d'accès aux différents emplacements sont sujettes à des codes spatiaux mis en place de manière spontanée. Cela crée un régime d'assignation des places par des groupes d'acteurs spécifiques: les touristes/photographes. Avec la multiplication des appareils numériques transportés par les touristes, il y a également une accumulation de photographies prises et, par conséquent, une mobilisation plus longue de certains emplacements particulièrement convoités par les autres. Le même plan peut en effet être pris par les différents appareils et il n'est pas rare d'entendre que plus de vingt clichés ont été pris selon une seule et même perspective. L'allongement de ce temps et donc de l'accaparation du spot engendre des stratégies différentes de gestion de l'espace. À titre d'exemple, la terrasse du Matterhorn Glacier Paradise, qui ne fait pas plus de quatre mètres sur cinq, offre un espace très restreint par rapport à sa fréquentation. Certaines personnes décident donc de fragmenter leurs séances de photographie. Pour ne pas accaparer ces spots, tant convoités, trop longtemps, ils laissent leur place pour revenir quelques instants plus tard et continuer leur activité. 
Se met ainsi en place une forme de circulation, cadencée par des moments de performances photographiques.

Sur les scènes photographiques, certains éléments du mobilier urbain - tels que les barrières, les plots, les bancs, les sculptures, les bacs à fleurs urbains et autres décors de la station - sont rapidement identifiés par les touristes comme supports à leur pratique, comme accessoires - objets nécessaires à la représentation et à la mise en scène de soi et du moment vécu. En parallèle, l'office du tourisme de la station a disposé - légèrement en dehors du pôle d'attractivité des photopoints - des objets dédiés expressément à l'usage de la photographie, tels que les sculptures ou les panneaux passe-tête. Ainsi, la logique de la pratique photographique des touristes ainsi que les mouvements des visiteurs dans la station ont été identifiés par les professionnels du tourisme et pris en compte afin de dynamiser l'espace: créer des espaces photographiques multipolaires et désengorger les photopoints.

Figure 1. Touristes posant en costumes d'époque devant le Cervin. Attraction photographique mise en place par l'office du tourisme

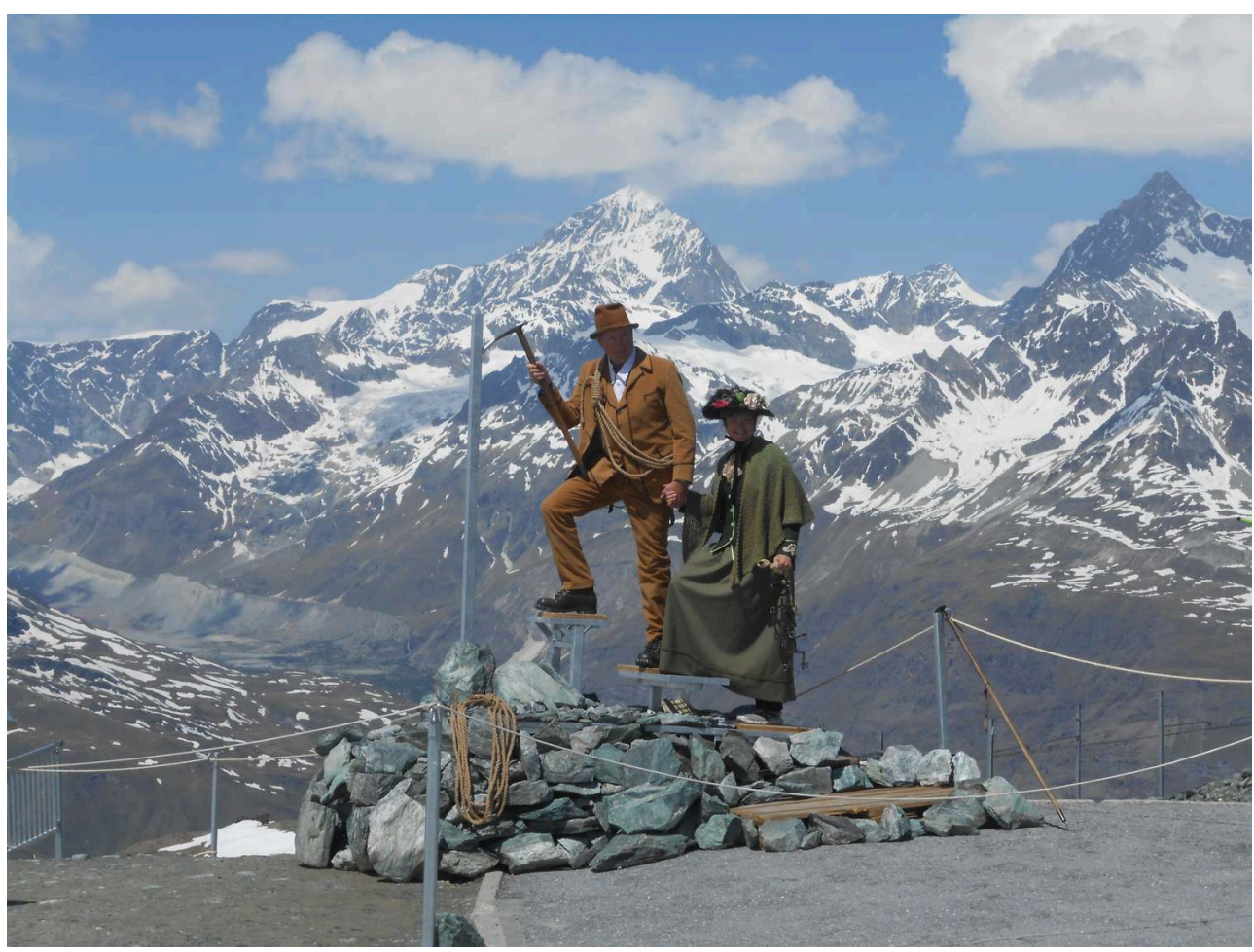

Entre scénographie et improvisation, ces chorégraphies, engendrées par le mouvement des différents éléments qui composent ces scènes photographiques, questionnent la manière d'appréhender et de conceptualiser les photopoints. Ces espaces illustrent de quelles manières les prescriptions et codes esthétiques liés à la photographie sont mis à l'épreuve des réalités vécues, de la coprésence d'autres êtres, objets ou codes. Dès lors, ils ne se donnent pas à voir comme des point fixes mais - pour reprendre les termes de Massey (2005) - comme des " constellations de processus » ou des " événements » en constant changement qui dépendent de la convergence des éléments qui les composent, à savoir : les touristes, les objets, l'appareillage numérique mais aussi les discours, les imaginaires, les anticipations ; évènements qui, par la suite, sont travaillés, triés et intégrés aux souvenirs qui nourrissent la trame de vie et l'histoire des 
individus. En ce sens, comme le montre Pink (2011), en s'inspirant des travaux d'Ingold (2007), ces espaces-évènements de la photographie sont à appréhender comme des enchevêtrements de trajectoires; trajectoires qui, durant les instants-photopoints, deviennent plus denses en coprésences.

Figure 2. Touriste photographiant le Cervin depuis un banc public

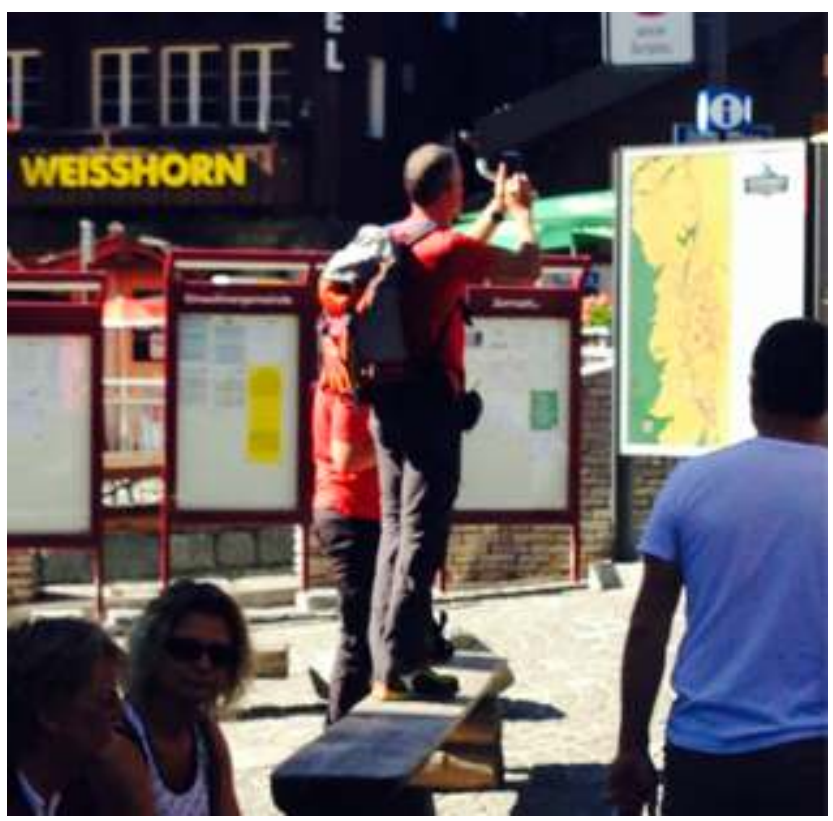

Investir de nouveaux espace-temps par la photographie

Figure 3. Train Glacier Express sur le trajet Visp-Zermatt

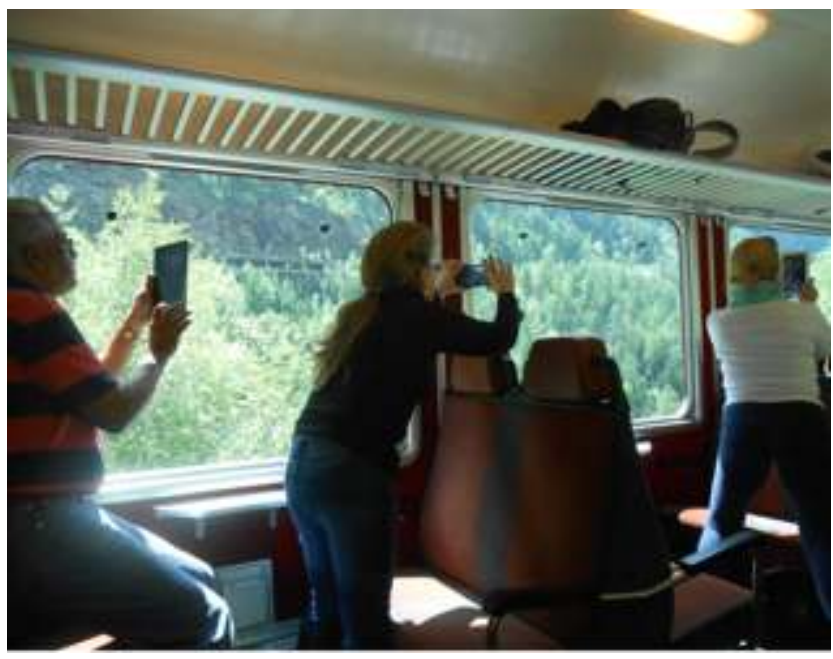


Figure 4. Affiche publicitaire dans le wagon du Glacier Express

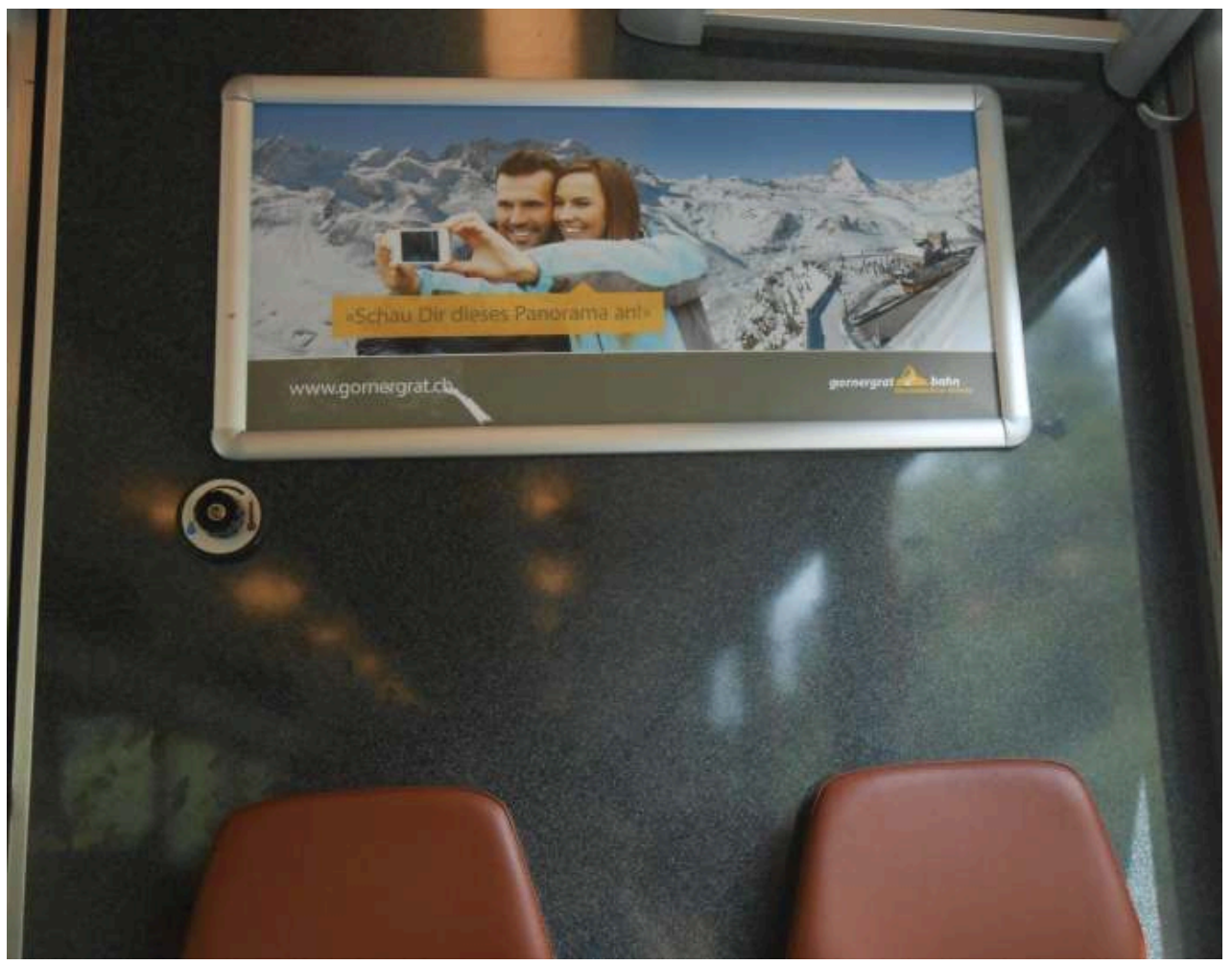

En entrant dans le wagon du Glacier Express, le train qui conduit les voyageurs à Zermatt, une affiche publicitaire de la même compagnie montrant un couple en train de se prendre en photo invite le voyageur à s'adonner à cette pratique ludique. À l'inverse de l'avertissement «appareils électroniques interdits » des avions, ici leur utilisation est non seulement acceptée mais encouragée. Et, en effet, une fois les premiers kilomètres parcourus et l'apparition des premiers signes de "paysage alpin", les voyageurs commencent progressivement à sortir leurs appareils. Au pluriel, car de manière générale l'appareillage des touristes présents est constitué de deux à trois appareils différents. Rapidement le wagon s'anime. Les corps se déplacent et les "clics" se joignent à l'espace sonore du train. Avec le déplacement des touristes de compartiment en compartiment, à la recherche de la "bonne place", les banquettes ou les appuie-tête - dont l'utilité première est de s'asseoir ou, comme leur nom l'indique, d'appuyer la tête - s'hybridifient et deviennent des trépieds ou autres supports de stabilisation de la photographie à venir. La contrôleuse qui, le temps d'un billet montré, interrompt ce spectacle ne fait aucun commentaire sur cet usage différencié des transports publics. Les différents appareils sont utilisés à tour de rôle pour photographier : une fois avec la tablette, une fois avec le smartphone, une fois avec l'appareil numérique reflex. Des tests se font et, le temps d'un instant, les appareils sont ajustés à la nature du paysage. Le rythme est soutenu. Le même paysage ne restant jamais longtemps devant la fenêtre, il faut être rapide et efficace en trouvant rapidement les bonnes stratégies de gestion de l'espace pour bien cadrer et adapter le meilleur mode de l'appareil photographique. Certaines personnes, faisant partie du même groupe, s'indiquent mutuellement, par un « oh, regarde ! ", les clichés à prendre. D'autres se montrent, et comparent par le biais de l'écran, les clichés pris. D'autres encore profitent d'un tunnel ou autre « temps mort " pour effacer quelques photographies considérées comme ratées. Le manège se termine quand le haut-parleur annonce en trois langues différentes l'arrivée du train en gare de Zermatt.

(Note de terrain sur le trajet Visp-Zermatt, juin 2015) 
Cette observation de terrain est représentative de la manière dont les potentialités du numérique ont permis à la pratique photographique de s'étendre à des espaces et des temps qui lui étaient jusqu'alors peu propices. Cette expansion a opéré des changements dans le rapport aux lieux, d'une fonctionnalité première assignée à certains lieux à l'investissement de ces derniers par la photographie. Si la photographie analogique était jusqu'alors fragmentée en moments phares distincts (le cadrage, la prise de vue, le développement des clichés et les moments de visionnage), les potentialités du numérique (augmentation du nombre de photographies, écran de visionnage, connexion à Internet) ont permis d'étendre la performance photographique à des espaces autres, tels que : les trains, télécabines, salles d'attente, chambres d'hôtel, bancs publics, mais également des espaces numériques online. "Je supprime les photos et choisis les meilleures quand je suis à la maison, ou dans le train, quand je ne fais rien de spécial», explique ainsi un jeune touriste allemand au Matterhorn Glacier Paradise. «Quand je n'ai rien à faire, je regarde les photos que j'ai prises, ça m'occupe (...), comme maintenant par exemple. Parfois, je les partage », m’a confié un autre touriste, assis sur un banc en attendant le prochain train pour le Gornergrat. Et c'est bien dans ce « quand je n'ai rien à faire » et « ça m'occupe » que se situe une des nouveautés amenées par le numérique dans le faire photographique. Outre les moments phares de la performance photographique, c'est-à-dire la capture du cliché, les touristes mobilisent les appareils photographiques en de nombreuses autres occasions. Durant le temps passé dans les trains, les télécabines, les salles d'attente, au restaurant en attendant leurs commandes, dans les chambres d'hôtel fréquentées le soir, sur les bancs publics - lors d'une pause dans le programme chargé de la journée -, les touristes se saisissent de leur outillage pour performer des types d'action très variés : vérifier la qualité des clichés, photographier, faire le tri des images, supprimer les photographies non satisfaisantes ou les partager via Internet. Comme le montre Löfgren (2008), malgré le discours présent dans les brochures promotionnelles portant sur l'extraordinaireté et l'intensité de l'expérience touristique vécue, le rythme du voyage est souvent parsemé de moments moins denses où attentes et rêveries occupent une place importante. Pour les touristes interrogés, ces temporalités se donnent à voir comme des temps entre-deux ou des temps libres, au sens où ils marquent un moment d'arrêt entre deux activités et entre deux espaces touristiques. Considérés comme inoccupés, ces instants ne semblent pas offrir, aux regards des visiteurs, de fonctions et de qualités touristiques suffisamment saillantes et, pour cela même, ils sont alors occupés par la photographie numérique. Parmi la palette de nouvelles activités rendues possibles par le numérique, il y a par exemple l'activité gestionnaire (consultation des archives photographiques, classification, gestion de la mémoire de l'appareil), l'activité communicationnelle (diffusion d'images sur les réseaux sociaux) ou l'activité de retouche d'images.

17 Ainsi, par le biais du numérique, la photographie s'est, d'une part, étendue à tout le séjour - et au-delà - et, d'autre part, elle a investi les moments non extraordinaires du tourisme en les rendant ludiques au même titre que d'autres activités proposées par la destination. 


\section{S'amuser avec la photographie : un relâchement contrôlé des corps}

En menant nos observations sur le terrain, il est rapidement apparu que l'amusement faisait partie intégrante du vécu photographique. Comme le souligne l'Équipe MIT (2005) :

L'histoire des pratiques touristiques est celle d'une affirmation du plaisir sous toutes ses formes : jeu, divertissement, sociabilité, mais aussi bien-être et libération des corps. (p. 270)

Or le numérique augmente ce divertissement. Larsen et Sandbye (2013) soulignent ainsi que le numérique pousse la photographie à être plus expérimentale et ludique, ce qui conduit à un certain relâchement des corps. Selon les auteurs, la gratuité et la jouissance du numérique peut partiellement expliquer la prolifération de cette pratique.

Sur l'ensemble des instants où les touristes s'emparent de leur outillage numérique, les moments de plaisir interviennent sous différentes formes. Ainsi, le trajet de retour en train jusqu'à Viège peut être transformé en moment de visionnage et de partage avec ses proches, où chacun, muni de son appareil photographique, prend grand soin à choisir la meilleure image pour la partager avec les autres. Mais c'est principalement lors de la prise de vue et du cadrage que la dimension ludique est la plus saillante: adopter des postures corporelles des plus variées - sauter, s'accroupir, faire la roue, créer des effets d'optiques en faisant tenir le Cervin sur la paume de la main (voir figure 4) - sont autant d'engagements corporels et affectifs du registre du loisir. En période hivernale, certains touristes s'amusent avec les boules de neige, d'autres construisent des bonhommes de neige et posent à leurs côtés. Pour optimiser ses chances de prendre le cliché souhaité par un minimum d'essais, les touristes parcourent les modes de leurs appareils pour trouver le plus compatible à la situation. 
Figure 5. Touriste se photographiant en face du Cervin

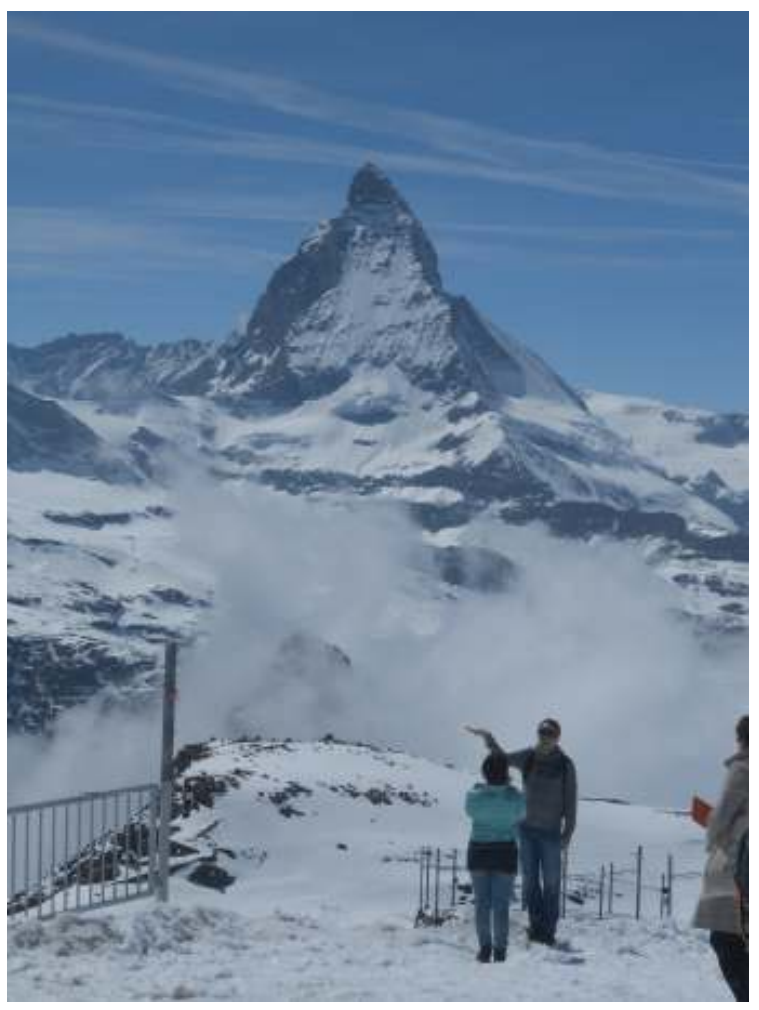

21 Jeanne, une femme française d'une trentaine d'années, est venue à Zermatt avec son compagnon pour le week-end. Sur la plateforme du Gornergrat, le couple a souhaité avoir un cliché d'eux en train de sauter devant le Cervin. Pour ce faire, Jeanne a demandé à des inconnus de prendre des clichés. Cette performance exigeait de leur part de sauter simultanément et, de la part du photographe, une grande concentration (voir figure 5). Après une dizaine d'essais, le cliché était dans la boîte. Interrogée sur ce moment, Jeanne a répondu :

C'est vrai qu'on a peut-être l'air un peu ridicules à sauter dans tous les sens, mais bon, l'endroit 臨le Gornergrat熙 est joli et on est en vacances. Et puis il y a une vue absolument magnifique sur les montagnes et le Cervin. Alors... on fait un peu les fous. 
Figure 6. Séquence d'une performance photographique face au Cervin

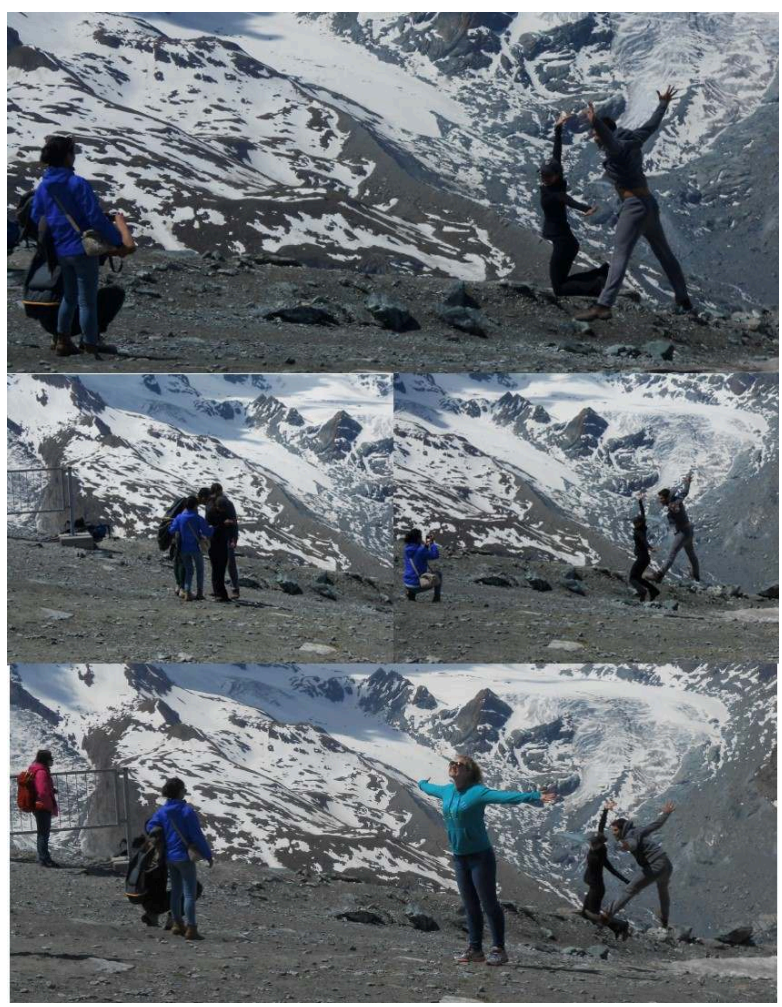

Avec cet exemple, nous sommes loin des postures figées et des corps raides devant se tenir immobiles le temps requis par la technicité de l'appareil. Le numérique permet de capturer le mouvement et cela conduit à des formes de relâchement corporel. Il n'en reste pas moins qu'il s'agit d'une mise en scène qui tend à une certaine représentation de soi guidée par des normes et codes sociaux - ceux du loisir, du plaisir et de la liberté. Dans cette recherche méticuleuse de la mise en scène de soi, du corps et des objets environnants, certaines normes de comportement, qui ne seraient pas admises dans d'autres contextes sociaux, sont acceptées. Cette liberté caractéristique des espacetemps de loisir se traduit par ce qu'Elias et Dunning (1986) nomment un relâchement contrôlé des contraintes. Ces instants sont également des instants solennels de «l'être ensemble ", qui expriment un sentiment d'intimité, de joie et de fête. Pour Bourdieu (1965) - qui a été l'un des premiers à accorder une importance à la dimension sociale de la photographie en sciences sociales -, tout comme les fêtes ou autres cérémonies ritualisées, les vacances sont des temps forts de la vie collective et surtout de la vie familiale. Pour lui, prendre en photo ou prendre la pose face à l'appareil renvoie à éterniser les moments culminants de la vie collective. Se mettre en scène a donc pour fonction sociale de

solenniser et d'éterniser les grands moments de la vie familiale, bref, de renforcer l'intégration du groupe familial en réaffirmant le sentiment qu'il a de lui-même et de son unité. (Bourdieu, 1965, p. 41) ${ }^{3}$

\section{Accommoder les restes de voyage}

Si l'on considère que ces espace-temps photographiques sont définis par un agrégat de sens et de récits qui y sont investis (Cresswell, 2003), quelles empreintes laissent-ils et par quel biais continuent-ils d'exister après le voyage, dans la trame des existences des 
individus? Que deviennent ces événements-photographiques une fois introduits dans la quotidienneté post-voyage. Une fois le cliché capturé, se pose donc la question de sa continuité. Encore faut-il que ces clichés continuent d'exister. Or, de nombreux touristes ont confié supprimer rapidement une grande partie de leur stock de photographies.

Avec le passage à la numérisation et la surabondance des images produites, de nombreuses photographies n'atteignent pas ce stade de maturation et sont supprimées rapidement après leurs captures. "Je les supprime probablement quand j'arrive à la maison. Je les télécharge dans l'ordinateur, puis je les parcours, j'en améliore quelquesunes si nécessaire. Mais les images que je n'aime pas, pouf, je les supprime », nous a ainsi confié un homme rencontré à la terrasse du Gornergrat. Si, avec l'analogique, les clichés des touristes s'assuraient une certaine pérennité en tant qu'objet matériel sur support papier, l'image sur écran ne bénéficie pas du même régime de permanence. La possibilité de prendre une quantité importante de photographies permet de choisir les images et les souvenirs à sauvegarder. Alors que déchirer une photographie papier d'un être cher reste une action difficile symboliquement, supprimer en un clic certains clichés numériques non convaincants ne semble pas provoquer de cas de conscience particulier chez les touristes interrogés. Pour beaucoup d'interviewés, une fois rentrés dans un environnement familier, l'étape du tri et du visionnage sur un écran plus grand constitue un rituel qui se réalise très rapidement après le voyage. Ces phases de sélection et de suppression - liant voyage et quotidien-contribuent à la transformation cognitive de l'ensemble de l'expérience et de l'émotion vécue durant le séjour en une mémoire choisie qui se cristallise et qui intègre une certaine forme de capital touristique des personnes. Comme le dit Bourdieu :

Les images du passé rangées selon l'ordre chronologique, «ordre de raison » de la mémoire sociale, évoquent et transmettent le souvenir des événements qui méritent d'être conservés [...]. (1965, p. 53)

Les touristes, prenant le rôle d'historiographe de leurs propres histoires, se plongent dans les souvenirs fraîchement créés et tissent des narrativités visuelles qui, par la suite, raconteront le séjour à Zermatt. Au cours de cette étape, où la masse d'images est retravaillée et transformée, la photographie change de statut, passant d'un outil de jeu, d'enchantement et de créativité à un objet de mémoire investi comme souvenir. Ainsi, la destruction progressive des objets photographiques fait partie intégrante du processus de mise en mémoire post-voyage. Et la possibilité de prendre un grand nombre de clichés photographiques donne une certaine liberté quant au choix des images gardées ou supprimées et donc au choix du récit de sa propre histoire et de sa propre mémoire.

26 Certaines photographies, à défaut d'être détruites, sont rangées. L'une des façons la plus standard d'accommoder ces restes de voyages consiste à les réunir en collections. Par le biais d'albums photos, les éléments du monde extérieur et de l'ailleurs de vacances sont introduits et traduits dans un espace privé et familier. Comme l'a montré Chambers (2002) en travaillant sur les albums de famille, ces collections - qui jouent le rôle de catalyseur - créent un dialogue visuel entre espace public et privé, entre un ailleurs de loisir et un quotidien du foyer familial, et entre une expérience touristique et son souvenir. Elles constituent des lieux de mémoire à une échelle individuelle ou familiale. Malgré les nombreux changements apportés par le numérique, le principe de l'album conserve une certaine continuité. Il réapparaît toutefois sous des formes 
matérielles nouvelles. De nombreux touristes interrogés disent qu'une fois de retour dans leur vie de tous les jours, ils créent des collections sur de nombreux espaces numériques : ils utilisent des logiciels qui proposent des espaces de classement (Iphoto, Picasa, Dropbox) disponibles en ligne et/ou hors ligne. L'ensemble de ces nouvelles fonctionnalités démultiplient les manières de raconter et de construire l'histoire visuelle de son voyage.

\section{Partager les photographies en ligne}

Les potentialités de diffusion en ligne des images fraîchement capturées constituent une autre part non négligeable du plaisir de photographier. Au moment de prendre le cliché, beaucoup de touristes ont confié penser aux futurs commentaires positifs qu'engendrera la photographie postée sur les réseaux sociaux numériques. Cet usage d'Internet pour la diffusion des images fait désormais partie du registre du quotidien et de la familiarité. Malgré les grandes potentialités d'Internet, il existe chez les touristes interrogés des modes d'usage très routiniers, qui se limitent à une série d'actions déterminées: consultation de leur courriel, envoi de messages ou de fichiers multimédias, échanges sur les réseaux sociaux. Toutes ces activités communicationnelles sont également faites durant le séjour touristique. La majorité des touristes interviewés disent aimer partager leurs photographies de voyage sur Internet avec leurs proches. Pour désigner les destinataires des photographies, les termes de cercle proche, amis proches, amis intimes, famille sont régulièrement employés par les interviewés. Or, à l'ère d'une société en réseau, dans laquelle le lien social n'est plus défini uniquement par la proximité géographique (Castells, 1996), la perception des touristes de ce qui est considéré comme le cercle proche est remise en question.

En outre, malgré le plaisir procuré par ce partage, il y a en contrepartie, chez certains individus, une crainte de la non-maîtrise de leur propre image. Voilà cinquante ans, Bourdieu a écrit : « Technique privée, la photographie fabrique des images privées de la vie privée » $(1965$, p. 53). Si la dimension privée demeure une dimension centrale des pratiques photographiques des amateurs, elle est néanmoins remise en cause par les espaces de diffusion numérique. Alors qu'avec l'analogique la photographie papier était relativement sédentaire et gardée dans des espaces connus et maitrisables, une fois transformée en code binaire et diffusée dans les espaces numériques, elle est devenue nomade et, par là même, transgressive. Avec la convergence technologique, qui permet à de nombreux appareils de "lire » les images numérisées, les photographies peuvent désormais naviguer à travers les frontières des différents espaces numériques. Du fait de cette itinérance entre les cartes mémoires, les ordinateurs, les blogs, les réseaux sociaux numériques, est apparue une incertitude quant à la délimitation des marges qui définissent ces espaces et, surtout, une crainte quant à la non-traçabilité et la nonmaîtrise de ses propres photographies.

Dans ces espaces numériques, où les frontières entre vie privée et vie publique, entre contrôlable et incontrôlable restent floues, se mettent en place des stratégies de maintien de ce qui est considéré comme étant sa vie privée. Parmi les touristes interviewés, l'utilisation des espaces de diffusion en réseau est sujette à une différenciation. Dans les discours des touristes à Zermatt, la diffusion sur les réseaux sociaux numériques ne dispose pas d'un capital de confiance aussi grand que la diffusion sur les messageries, type WhatsApp. «WhatsApp, je préfère. C'est rapide, 
gratuit [...]. Généralement, je l'envoie à un groupe de deux à trois personnes. Comme ça, il n'y a pas tout le monde qui peut le voir » (femme, suisse, 35 ans). Autre stratégie, il arrive que seuls quelques éléments personnels apparaissent sur l'image : les pieds, une peluche, un dos, une ombre... Cette manière détournée de se mettre en scène, sans passer par l'inscription de son corps entier dans l'image, permet de le faire tout en préservant un certain anonymat.

Cette perception de ce qui constitue un espace public ou privé de diffusion, sécurisé ou non, est individuelle et dépend du contexte, du milieu socio-culturel et des compétences techniques des individus. Ces éléments influencent la manière dont les outils numériques sont manipulés ou, au contraire, évités.

\section{En conclusion}

L'argument central de cet article est de présenter le faire photographique numérique comme un agrégat de temps sociaux et un ensemble de pratiques qui imbriquent: matérialité numérique de l'appareillage photographique; corporalité, à travers des techniques, des postures, des gestes ; affect, à travers les émotions, les imaginaires et les considérations esthétiques; et des spatialités touristiques. À travers l'analyse des microinstants qui forment cet ensemble hétérogène que constitue la photographie, cet article a tenté de montrer que les temporalités touristiques de la photographie, par le biais du numérique, se sont, d'une part, étendues à tout le séjour - et au-delà - et, d'autre part, qu'elles ont investi les moments non extraordinaires du tourisme en les rendant ludiques au même titre que d'autres activités proposées par la destination. Cette manière d'envisager une pratique touristique comme un ensemble d'agrégats hétérogènes qui se déploient sur des temporalités étendues peut être appliquée à d'autres types d'activités touristiques. À titre d'exemple, de même que la photographie ne se limite pas aux instants de capture d'images, la pratique du ski ne se limite pas aux moments passés sur les pistes, les pratiques balnéaires ne se limitent pas à celle de la plage, ou encore la randonnée à l'unique acte de marcher. Enfin, le numérique étant présent de manière transversale au sein de nombreuses activités touristiques, il semble nécessaire de prêter attention aux éventuelles métamorphoses apportées par ce dernier afin de rendre compte de l'état actuel des pratiques en question.

\section{BIBLIOGRAPHIE}

Pierre BOURDIEU, Un art moyen. Essai sur les usages sociaux de la photographie, éditions de Minuit, 1965.

Michel CASTELLS, The Rise of the Network Society, Blackwell, 1996.

Deborah CHAMBERS, « Family as Place: Family Photograph Albums and the Domestication of Public and Private Space ", dans Joan SCHWARTZ et James RYAN (dir.), Picturing Place: Photography and the Geographical Imagination, I.B. Tauris, 2002. 
Mike CRANG, «Picturing practices: research through the tourist gaze », Progress in Human Geography, vol. 21, n³, 1997.

Tim CRESSWELL, «Theorizing place, mobilizing place, placing mobility: the politics of representation in a globalized world », Thamyris/Intersecting: Place, Sex and Race, vol. 21, 2003.

David CROUCH et Luke DESFORGES, « The sensuous in the tourist encounter: introduction: the power of the body in Tourist Studies », Tourist Studies, vol. 3, n 1, 2003.

David CROUCH et Nina LÜBBREN, Visual culture and tourism, Berg, 2003.

Thomas J. CSORDAS, Embodiment and Experience: The Existential Ground of Culture and Self, Cambridge University Press, 1994.

Robert R. DESJARLAIS et Jason THROOP, «Phenomenological Approaches in Anthropology ", Annual Review of Anthropology, vol. 40, n 1, 2011.

Norbert ELIAS et Eric DUNNING, Sport et civilisation. La violence maîtrisée, Fayard, 1986.

Équipe MIT, Tourisme 2. Moments de lieux, coll. « Mappemonde », Belin, 2005.

Michael HALDRUP et Jonas LARSEN, « Reading of tourist photographs », dans Deborah CHAMBERS et Tijana RAKIC (dir.), An introduction to visual research methods in tourism, Routledge, 2012.

Tim INGOLD, Lines: A brief history, Routledge, 2007.

Nancy A. VAN HOUSE, «Personal photography, digital technologies and the uses of the visual », Visual Studies, vol. 26, n², 2011.

Jonas LARSEN, « (Dis)Connecting Tourism and Photography: Corporeal Travel and Imaginative Travel », Journeys: International Journal of Travel and Travel Writing, vol. 5, n² 2, 2004.

Jonas LARSEN, Performing tourist photography, thèse de doctorat (non publiée), Université de Roskilde, 2003.

Jonas LARSEN et Mette SANDBYE, Digital Snaps: The New Face of Photography, I.B. Tauris, 2013.

Naomi LEITE et Nelson H.H. GRABURN, «L'anthropologie pour étudier le tourisme », Mondes du Tourisme, vol. 1, 2010.

Orvar LÖFGREN, « The secret lives of tourists: Delays, disappointments and daydreams ", Scandinavian Journal of Hospitality and Tourism, vol. 8, $\mathrm{n}^{\circ} 1,2008$.

Michel LuSSAUlt, De la lutte des classes à la lutte des places, Grasset, 2009.

Doreen MASSEY, For space, Sage, 2005.

Peter OSBORNE, Travelling Light: Photography, Travel and Visual Culture, Manchester University Press, 2000.

Sarah PINK, « Amateur photographic practice, collective representation and the constitution of place », Visual Studies, vol. 26, n² 2, 2011.

Mike ROBINSON et David PICARD, « Moments, magic and memories: Photographing tourists, tourist photographs and making worlds », dans Mike ROBINSON et David PICARD (dir.), The framed world: Tourism, tourists and photography, Ashgate, 2009.

Joanna SASSOON, « Photographic Materiality in the Age of Digital Reproduction », dans Elizabeth EDWARDS et Janice HART (dir.), Photographs Objects Histories: On the materiality of images, Routledge, 2004. 
Caroline SCARLES, «Becoming tourist: Renegotiating the visual in the tourist experience »,

Environment and Planning D: Society and Space, vol. 27, n 3, 2009.

Jonathan SCHROEDER, Visual Consumption, Routledge, 2002.

Susan SONTAG, On photography, Penguin Books, 1977.

John TAYLOR, A Dream of England: Landscape, Photography and the Tourist's Imagination, Manchester University Press, 1994.

John URRY, The Tourist Gaze, $2^{\mathrm{e}}$ édition, Sage, 2002.

John URRY, The tourist gaze: leisure and travel in contemporary societies, Sage, 1990.

Soile VEIJOLA et Eeva JOKINEN, « The Body in Tourism », Theory, Culture \& Society, vol. 11, n 3, 1994.s

\section{NOTES}

1. Traduction française de l'auteure. Dans l'article original, en anglais, le terme est « existential null point » (Desjarlais et Throop, 2011, p. 89).

2. Voir : https://www.zermatt.ch/fr/Carte-Interactive/Curiosites/Photopoints/Pointsphotographiques

3. Si, dans son ouvrage, Bourdieu met l'accent sur la fonction familiale de la photographie et la réaffirmation solennelle de son unité de groupe, cette dominance de la dimension collective se doit d'être repensée à l'aune de l'apparition du numérique et de la diversification des configurations de l'institution familiale.

\section{RÉSUMÉS}

Alors que le numérique a apporté des changements notoires dans les manières de faire de la photographie par les touristes, les recherches prenant en compte cette nouvelle dimension dans l'analyse des pratiques photographiques restent rares. Fondé sur une enquête ethnographique menée auprès de touristes visitant la station suisse de Zermatt, cet article vise à comprendre les différentes dimensions du faire photographique en apportant un éclairage sur les manières dont les touristes s'approprient, négocient et incorporent les nouvelles potentialités numériques durant leurs performances photographiques. À travers une description détaillée des différents microinstants qui séquencent et rythment la pratique photographique numérique des touristes à Zermatt, la photographie est ici envisagée comme un ensemble de pratiques sociales, incorporées et réflexives, qui associent normes sociales, codes esthétiques, dispositifs techniques numériques, corporéité, sensorialité et espaces, et ce d'une nouvelle façon et sur des temporalités allant audelà de l'unique instant de la capture d'image.

As digitalization has brought major transformations to the way individuals engage with photography, research focusing on tourist practices and performances has often disregarded the essential dimension of technology. Based on an ethnographical account of tourists' behaviours in the Swiss ski resort Zermatt, this paper aims to understand different dimensions of the photographic doing by drawing attention to the way tourists master, negotiate and embody new 
digital potentialities during their photographic performances. Throughout a detailed description of the micro-instants that rhythm tourists' digital photographical practices, this analysis conceptualizes contemporary photography as an aggregate of embodied and reflexive practices, which encompass social norms, aesthetic codes, embodied sensory practices, digital materialities and space.

\section{INDEX}

Mots-clés : photographie numérique, pratiques touristiques, performance, Zermatt Keywords : digital photography, touristic practices, performance, Zermatt

\section{AUTEUR}

\section{ELLINA MOURTAZINA}

Assistante-doctorante, Institut de géographie et durabilité, Université de Lausanne, Anthropologie du tourisme ellina.mourtazina@unil.ch 